\title{
Spin-split subbands and magneto-oscillations in III-V asymmetric heterostructures
}

\author{
E. A. de Andrada e Silva, * G. C. La Rocca, and F. Bassani \\ Scuola Normale Superiore, Piazza dei Cavalieri 7, I-56100 Pisa, Italy
}

(Received 2 May 1994)

\begin{abstract}
A quantum-mechanical study of the magneto-oscillations in asymmetric heterostructures is presented with the aim of clarifying the origin and the effects of the spin-orbit spin splitting in the conduction subband. The magnetization of the two-dimensional electron gas at the interface of modulation-doped III-V semiconductor heterojunctions is calculated as a function of applied magnetic field and carrier concentration, taking into account both spin-orbit (zero-field) spin-splitting contributions: one due to the $k^{3}$ bulk term and one due to the lack of specular symmetry $\sigma_{h}$ along the growth direction. Regular beating patterns in the amplitude of the oscillations are shown to originate from the latter term. The $k^{3}$ term introduces a $k$-space anisotropy in the zero-field spin splitting. This leads to anomalous beating patterns, related to the occurrence of a magnetic breakdown at special points of the Fermi surface with a small spin splitting. Experimental evidence of regular beating patterns has been found in InAs-based heterostructures. The possibility of observing anomalous beating patterns in $\mathrm{GaSb}$ heterojunctions is discussed.
\end{abstract}

\section{INTRODUCTION}

Time-reversal symmetry at zero external magnetic field guarantees the spin degeneracy of the electronic states only in the presence of spatial inversion symmetry. The spin splitting in the conduction band of zinc-blende semiconductor compounds, due to inversion asymmetry, has been predicted long ago, ${ }^{1}$ and detected with different measurements, ${ }^{2}$ one of its characteristic manifestations being a beating pattern in the amplitude of the magnetooscillations. ${ }^{3,4}$ The conduction subbands in asymmetric quantum wells are also spin split by the spin-orbit coupling and a clear beating pattern in the Shubnikov-de Haas data has been observed in such systems. ${ }^{5-7}$ The question of whether the spin splitting comes from the inversion asymmetry in the bulk potential $\left(k^{3}\right.$ term) or from the lack of specular symmetry in the quantum well (Rashba term ${ }^{8}$ ) has however found no definite answer. This was in part due to the lack of comparison with complete model calculations. In this paper, we obtain, through a full quantum-mechanical calculation, the beating pattern in the magneto-oscillations from a model Hamiltonian, which includes the two above-mentioned spin-splitting terms and nonparabolicity corrections as well.

The de Haas-van Alphen and Shubnikov-de Haas effects are example of general magneto-oscillations, which are a pure quantum-mechanical effect due to the Landau energy quantization in the presence of a magnetic field. ${ }^{9}$ Such oscillations have been extensively used to determine the electronic properties of bulk semiconductors and metals (Fermi-surface geometry, effective masses, etc.). ${ }^{10}$ Magneto-oscillations observed in $\mathrm{Si}$ inversion layers have been the first evidence of the existence of a two-dimensional electron gas, ${ }^{11}$ and continue to be a very important tool in the study of these systems. Here we are concerned with the effects of the spin splitting on the magneto-oscillations.
Each discrete Landau level presents a degeneracy $g=e B / \pi \hbar$ per unit area, including spin degeneracy, where $B$ is the perpendicular magnetic-field strength. With increasing field intensity, the electrons are redistributed among a decreasing number of levels. The number of occupied levels at zero temperature is $n=n_{s} / g$, where $n_{s}$ is the carrier concentration per unit area. It changes by one whenever the inverse of the field changes by $\Delta(1 / B)=e / n_{s} \pi \hbar$. When only the lowest subband is occupied [pure two-dimensional (2D) case], $n_{s}=2 A /(2 \pi)^{2}$, where $A$ is the area in $\mathbf{k}$ space filled by the 2D Fermi see, and the above period gives the celebrated semiclassical Onsager expression for the period of the magnetooscillations. ${ }^{12}$ Measurements of this period are commonly used to determine the surface carrier density $n_{s}$. Inclusion of the spin-orbit interaction modifies the above picture by introducing the zero-field spin splitting and by correcting the Zeeman coupling.

The spin splitting in the conduction subband of asymmetric heterostructures is due to contributions from both mechanisms, bulk $k^{3}$ and Rashba asymmetry, ${ }^{13}$ but their relative significance has not been sufficiently investigated. It appears that modulation-doped semiconductor heterojunctions with intermediate gap III-V semiconductor compounds, like InAs and GaSb, are systems of particular interest to study the competition between them. In this case, the degree of specular asymmetry of the confining well is given by the electric field at the interface. By changing it, one can vary the relative contribution of the two splitting mechanisms. The spin splitting becomes strongly anisotropic as the two mechanisms become comparable, ${ }^{14}$ and we will show that this leads to anomalous beating patterns in the magneto-oscillations. Here we will focus on heterojunctions based on InAs and $\mathrm{GaSb}$, grown on appropriate larger gap materials.

In Sec. II, a model for semiconductor heterojunctions is presented in some detail and applied to both types of heterojunctions. The results obtained for the oscillations 
in the magnetization are presented and discussed in Sec. III. Conclusions are presented in Sec. IV.

\section{MODEL FOR SEMICONDUCTOR HETEROJUNCTIONS}

We consider heterojunctions in a range of carrier concentrations where only the lowest subband is occupied. In such a case, a good model consists of independent electrons in the III-V semiconductor compound, confined by a triangular potential well formed by the constant electric field $E=e n_{s} / \epsilon_{\mathrm{sc}}$, along the growth direction, plus an infinite potential barrier at the interface with a much larger band-gap material (see Fig. 1). The carrier concentration $n_{s}$ is equal to that of ionized donors in the barrier and $\epsilon_{\mathrm{sc}}$ is the semiconductor dielectric constant.

The semiconductor compound is described by the eight-band $\mathbf{k} \cdot \mathbf{p}$ Kane model and the solution for the lowest conduction subband is obtained within the multiband effective-mass envelope-function approximation. This is the simplest possible model containing the relevant physics for the study of the zero-field spin split-

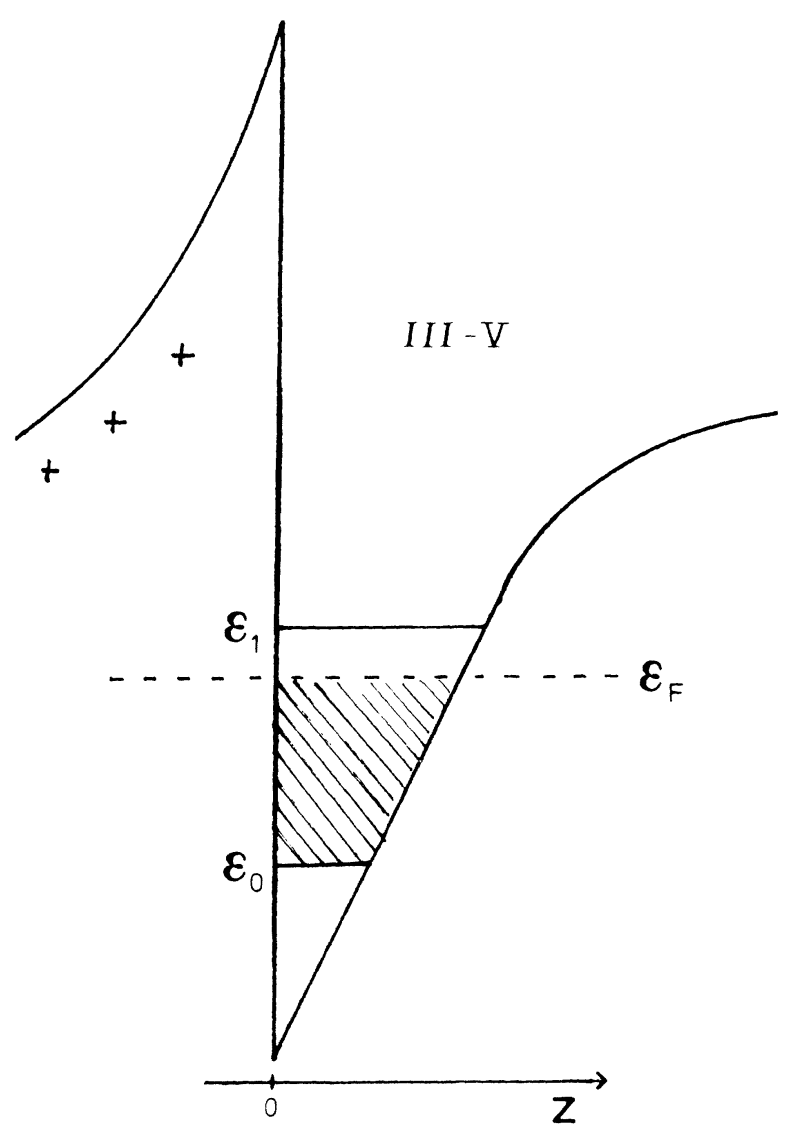

FIG. 1. Illustration of a modulation-doped semiconductor heterojunction. The lowest two subbands are indicated. The position of the Fermi level is determined by the occupancy of the propagating states along the plane. ting in asymmetric heterostructures and the beating pattern in the magneto-oscillations.

\section{A. Zero-magnetic field}

We shall first consider the heterojunction in the absence of any external magnetic field. The solution at zero field helps to elucidate the structure and origin of the $\mathbf{k}$ dependent spin splitting in the first subband and is used in the semiclassical analysis of the magneto-oscillations. First, the eight-band Kane Hamiltonian is projected into the $2 \times 2$ conduction-band space, and expressions for the nonparabolicity and Rashba terms are derived. The bulk $k^{3}$ term, originating from interactions with remote bands, will be considered separately.

\section{Rashba spin-orbit spin splitting}

We start from the Kane model. ${ }^{15}$ Let $S, X, Y$, and $Z$ denote the conduction- and the tree-valence bulk Bloch functions at the zone center. As basis functions, we choose the following linear combinations, which have been shown to be particularly convenient for the heterostructure problem ${ }^{16}$

$$
\begin{aligned}
& u_{1}=S \uparrow, \\
& u_{2}=\sqrt{\frac{2}{3}} Z \uparrow+\frac{1}{\sqrt{6}}(i X \uparrow+Y \downarrow), \\
& u_{3}=-\frac{1}{\sqrt{2}}(-i X \uparrow+Y \downarrow), \\
& u_{4}=-\frac{1}{\sqrt{3}}[Y \downarrow-(Z-i X) \uparrow], \\
& u_{5}=-S \downarrow, \\
& u_{6}=-\sqrt{\frac{2}{3}} Z \downarrow+\frac{1}{\sqrt{6}}(i X \downarrow+Y \uparrow), \\
& u_{7}=-\frac{1}{\sqrt{2}}(-i X \downarrow+Y \uparrow), \\
& u_{8}=-\frac{1}{\sqrt{3}}[Y \uparrow+(Z+i X) \downarrow] .
\end{aligned}
$$

The arrows indicate the spin state with respect to the $y$ axis. Making use of the spherical symmetry of the Kane model, we choose the parallel wave vector $\mathbf{k}$ along the $x$ axis and the growth direction along the $z$ axis. The electron wave function will then be given by

$$
\psi(\mathbf{r})=e^{i k x} \sum_{j=1}^{8} f_{j}(z) u_{j}(\mathbf{r})
$$

where the $f_{j}$ are the envelope functions, and the effective-mass Hamiltonian is block diagonalized,

$$
H=\left(\begin{array}{cc}
H_{+} & 0 \\
0 & H_{-}
\end{array}\right),
$$

with 


$$
H_{ \pm}=\left(\begin{array}{cccc}
V(z) & P\left(\frac{d}{d z} \mp \frac{k}{2}\right) & \mp \frac{\sqrt{3}}{2} P k & \frac{P}{\sqrt{2}}\left(\frac{d}{d z} \pm k\right) \\
P\left(-\frac{d}{d z} \mp \frac{k}{2}\right) & V(z)-E_{g} & 0 & 0 \\
\mp \frac{\sqrt{3}}{2} P k & 0 & V(z)-E_{g} & 0 \\
\frac{P}{\sqrt{2}}\left(-\frac{d}{d z} \pm k\right) & 0 & 0 & V(z)-E_{g}-\Delta
\end{array}\right),
$$

where $V(z)$ is the confining potential, the momentum matrix element $P=\sqrt{\frac{2}{3}} \hbar / m_{e}\left\langle i S\left|p_{x}\right| X\right\rangle\left(m_{e}\right.$ being the bare electron mass), $E_{g}$ is the band gap, and $\Delta$ is the spin-orbit energy splitting. The $k^{2}$ free particle term has been neglected in the diagonal matrix elements because the small electron effective mass guarantees that the dispersion is given by the off-diagonal contribution.

So far, we have just extended the scheme introduced by Marques and Sham ${ }^{16}$ in order to include the split-off bands. The split-off bands are, however, very important in determining the spin-dependent effects in the conduction band. This is indicated, for example, by the comparison of the effective electron $g^{*}$ factor as given by the models with six and eight bands and the measured ones. Table I shows how big an improvement one gets by adding the split-off bands.

The multiband effective-mass equation with Hamiltonian (3) leads to two sets of four coupled equations for the two groups of envelope functions. Eliminating the other components, we arrive at the following Schrödinger-like equation for the conduction-band envelope functions:

$$
\begin{aligned}
{\left[-\frac{\hbar^{2}}{2} \frac{d}{d z} \frac{1}{m(z, \varepsilon)} \frac{d}{d z}\right.} & +\frac{\hbar^{2} k^{2}}{2 m(z, \varepsilon)} \\
& +V(z) \mp \alpha(z, \varepsilon) k-\varepsilon] f_{ \pm}=0
\end{aligned}
$$

$\left(f_{+}=f_{1}\right.$ and $\left.f_{-}=f_{5}\right)$ where $\varepsilon$ is the electron energy,

$$
\frac{1}{m(z, \varepsilon)}=\frac{P^{2}}{\hbar^{2}}\left(\frac{2}{\varepsilon-V(z)+E_{g}}+\frac{1}{\varepsilon-V(z)+E_{g}+\Delta}\right)
$$

and

$$
\alpha(z, \varepsilon)=\frac{P^{2}}{2} \frac{d}{d z}\left(\frac{1}{\varepsilon-V(z)+E_{g}}-\frac{1}{\varepsilon-V(z)+E_{g}+\Delta}\right) .
$$

TABLE I. Electron effective $g^{*}$ factor as given by different k·p models. The bulk parameters used are given in Table II.

\begin{tabular}{lccr}
\hline & $6 \times 6^{\mathrm{a}}$ & $8 \times 8^{\mathrm{b}}$ & Expt. $^{\mathrm{c}}$ \\
\hline InAs & -41.5 & -14.4 & -14.7 \\
$\mathrm{GaSb}$ & -22.7 & -7.43 & -9.1 \\
\hline \hline
\end{tabular}

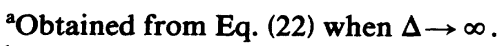

${ }^{\mathrm{b}}$ As given by Eq. (22).

${ }^{\mathrm{c}}$ Landolt-Börnstein tables.
The term linear in $\mathbf{k}$ gives the spin splitting. It is zero for the bulk $[V(z)=$ const $]$ and has expectation value equal to zero for symmetric quantum wells. Note that the Kane model gives no $k^{3}$ splitting, so that only the asymmetry of the quantum well contributes.

In order to solve Eq. (5), we make use of the fact that we are interested in the conducting states of the first subband, which have $\varepsilon$ (the energy measured from the bottom of the conduction band), much smaller than the energy parameters entering the model, i.e., $E_{g}$ and $\Delta$. A simple and particularly transparent solution is obtained expanding $1 / m(z, \varepsilon)$ and $\alpha(z, \varepsilon)$ in powers of ${ }^{19}$

$$
\delta=\frac{\varepsilon-V(z)}{E_{g}+\Delta}
$$

which is in fact, as shown below, for the bound states we are interested in, a very small parameter in most heterojunctions of interest. The zero-order term in the expansion gives the parabolic approximation,

$$
\left[\frac{\hbar^{2}}{2 m^{*}}\left[-\frac{d^{2}}{d z^{2}}+k^{2}\right]+V(z)-\varepsilon_{0}\right] f_{ \pm}=0
$$

with

$$
m^{*}=\frac{\hbar^{2}}{P^{2}} \frac{E_{g}\left(E_{g}+\Delta\right)}{3 E_{g}+2 \Delta},
$$

and no spin splitting. The zero-field spin-splitting appears only in first order in $\delta$, together with the nonparabolicity. The energy in the conduction subband can be written as

$$
\begin{aligned}
\varepsilon= & \left\langle f_{ \pm}\right| \frac{\hbar^{2}}{2 m^{*}}\left(\frac{d^{2}}{d z^{2}}+k^{2}\right]-a \frac{\left[\frac{\hbar^{2}}{2 m^{*}}\left[-\frac{d^{2}}{d z^{2}}+k^{2}\right]\right]^{2}}{E_{g}+\Delta} \\
& +V(z) \mp \alpha k\left|f_{ \pm}\right\rangle,
\end{aligned}
$$

where $a$, the nonparabolicity constant, is given by

$$
a=\frac{2\left(E_{g}+\Delta\right)^{2}+E_{g}^{2}}{E_{g}\left(3 E_{g}+2 \Delta\right)},
$$

and the spin-splitting parameter

$$
\alpha=\frac{\hbar^{2}}{2 m^{*}} \frac{\Delta}{E_{g}} \frac{2 E_{g}+\Delta}{\left(E_{g}+\Delta\right)\left(3 E_{g}+2 \Delta\right)} e E
$$

is identified with the Rashba spin-orbit coupling parame- 
ter in the case of a constant electric field $E[V(z)=e E z]$. This leads to an isotropic spin splitting given by $\Delta_{s}=2 \alpha k$. By letting $\Delta \rightarrow \infty$, we recover the six-band model expression for $\alpha$, obtained in Ref. 14. Allowing for different momentum matrix elements, i.e., using the experimental band-edge effective mass in both cases, we find that the Rashba spin-orbit coupling parameter in the eight-band model is reduced by a factor of $\sim 0.6$ with respect to that given by the six-band model, for both InAs and $\mathrm{GaSb}$.

Equation (11) contains explicit analytical expressions for the spin orbit and nonparabolicity corrections, which is an important simplification with respect to Eq. (5) where $m$ and $\alpha$ depend on the energy value $\varepsilon$. Equation (11) is amenable to a simple variational approach because the lowest subband of such a triangular well can be well described with the Fang-Howard trial function,

$$
f_{ \pm}(z)=\sqrt{b^{3} / 2} z e^{-b z / 2}
$$

where $b$ is determined by minimizing the total energy of all the electrons, which, assuming that the electric field is only due to the electrons themselves and given by $E=e n_{s} / \epsilon_{\mathrm{sc}}$, amounts to minimizing $\varepsilon^{\prime}=\varepsilon(k)-3 e E / 2 b$ (the last factor corrects the double counting in the e-e interaction ${ }^{20}$ ), with the spin-independent single-particle energy given by

$$
\begin{aligned}
\varepsilon(k)= & \frac{\hbar^{2}}{2 m^{*}}\left(\frac{b^{2}}{4}+k^{2}\right) \\
& -a \frac{\hbar^{4} / 4 m^{* 2}}{E_{g}+\Delta}\left(-\frac{3 b^{4}}{16}+\frac{b^{2} k^{2}}{2}+k^{4}\right)+\frac{3 e E}{b} .
\end{aligned}
$$

The dispersion relation of the split subbands is given by $\varepsilon_{ \pm}(k)=\varepsilon(k) \mp \alpha k$. The value of the variational parameter $b$ will obviously depend on $k$. This is the well-known coupling between the motions perpendicular and parallel to the interface due to nonparabolicity. The dependence however is weak. In all the cases considered here, as $k$ increase from zero up to $k_{F}, b$ never changes by more than $10 \%$.

The above solution for the first conduction subband of a semiconductor heterojunction is seen to have kept the analytical simplicity of the one-band model, taking into account important effects as nonparabolicity and spinorbit spin splitting. We show in Fig. 2 specific examples in order to compare with the parabolic approximation. The values of the parameters used are listed in Table II. The accuracy of this approximation depends on the smallness of $\delta^{2}$, which increases with both $k$ and $n_{s}$. We have estimated it by computing its expectation value at $k_{F}$ (on the Fermi surface). For the largest carrier concentration we consider here, i.e., $n_{s}=10^{12} \mathrm{~cm}^{-2}$, we find $\delta_{\max }^{2} \simeq 0.003$ and $\simeq 0.03$, for GaSb- and InAs-based heterojunctions, respectively, so that the approximation adopted is fully justified, at least within $10 \%$ accuracy.

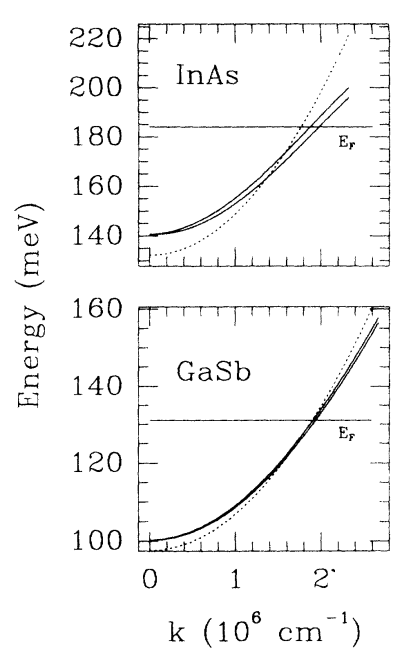

FIG. 2. Spin-orbit (Rashba) split lowest subbands with nonparabolicity in InAs- and GaSb-based heterojunctions, both with $n_{s}=6.0 \times 10^{11} \mathrm{~cm}^{-2}$. The Fermi energy and the parabolic approximation (dashed lines) are also shown. The zero of energy corresponds, in each case, to the bottom of the bulk conduction band.

\section{Bulk $k^{3}$ contribution}

As mentioned in the Introduction, another important contribution to the zero-field spin-splitting in III-V semiconductor compound quantum wells derives from the inversion asymmetry of the zinc-blende crystal structure of the host material. It is well known that, in lowest order in $k$, this splitting is described in the bulk by a term of the form ${ }^{21-23}$

$$
\begin{aligned}
H_{k^{3}}=\gamma[ & \sigma_{x} k_{x}\left(k_{y}^{2}-k_{z}^{2}\right) \\
& \left.+\sigma_{y} k_{y}\left(k_{z}^{2}-k_{x}^{2}\right)+\sigma_{z} k_{z}\left(k_{x}^{2}-k_{y}^{2}\right)\right],
\end{aligned}
$$

where $\sigma_{i}$ stands for the Pauli spin matrices, $\gamma$ is a material constant, and the coordinate axis are now assumed parallel to the crystallographic cubic axis. This $k^{3}$ splitting is much smaller than the quantized energy $\varepsilon$ of the confined electrons and its effects can be treated within first-order perturbation theory. ${ }^{14,24,25}$ In this case, all one has to do is to replace $k_{z}$ and $k_{z}^{2}$ by the expectation values of $-i d / d z$ and $-d^{2} / d z^{2}$, respectively, in the first unperturbed conduction subband.

TABLE II. Bulk parameters. Except for $\gamma$, the parameters we use, listed below, are the low-temperature figures in the Landolt-Börnstein tables.

\begin{tabular}{lccccc}
\hline \hline & $E_{g}(\mathrm{eV})$ & $\Delta(\mathrm{eV})$ & $m^{*}\left(m_{e}\right)$ & $\gamma\left(\mathrm{eV} \AA^{3}\right)$ & $\epsilon_{\mathrm{SC}}$ \\
\hline InAs & 0.418 & 0.38 & 0.023 & $130^{\mathrm{a}}$ & 12.2 \\
$\mathrm{GaSb}$ & 0.813 & 0.75 & 0.041 & $187^{\mathrm{b}}$ & 14.4 \\
\hline \hline
\end{tabular}

${ }^{\mathrm{a}}$ Estimated here, following Ref. 17.

${ }^{b}$ Reference 18. 


\section{Total zero-field spin splitting}

In our case, we can then use the expressions (11) and (16) to write the total spin Hamiltonian for an arbitrary direction of the two-dimensional $k$ vector using the Rashba symmetry argument. ${ }^{8}$ We obtain

$$
H_{\text {spin }}=\sigma \cdot(\gamma \kappa+\alpha \mathbf{k} \times \hat{z})
$$

where $\mathbf{k}$ is bidimensional, and

$$
\kappa=k_{x}\left(k_{y}^{2}-\frac{b^{2}}{4}\right) \hat{x}+k_{y}\left(\frac{b^{2}}{4}-k_{x}^{2}\right) \hat{y} .
$$

We denote the eigenvalues of $H_{\text {spin }}$ by $\pm \Delta_{s} / 2$, with the total spin splitting $\Delta_{s}$, given by

$$
\begin{gathered}
\Delta_{s}(k, \theta)=2\left[\left[\gamma^{2} \frac{b^{4}}{16}+\alpha^{2}\right] k^{2}+\gamma \alpha\left[k^{2}-\frac{b^{2}}{2}\right] k^{2} \sin 2 \theta\right. \\
\left.+\gamma^{2}\left(k^{2}-b^{2}\right) \frac{k^{4}}{4} \sin ^{2} 2 \theta\right]^{1 / 2}
\end{gathered}
$$

where $\theta$ is the angle between the parallel wave vector $\mathbf{k}$ and the $x$ axis of the cubic crystal. The total single-particle eigenenergy is given by $\varepsilon_{ \pm}(k, \theta)$ $=\varepsilon(k) \pm \Delta_{s}(k, \theta) / 2$. Note that the \pm spin states refer now to a direction of angular-momentum quantization, which depends on both $k$ and $\theta$, as indicated in Eqs. (17) and (18). We have plotted in Fig. 3 the total zero-field spin splitting $\Delta_{s}$ at three different points of the Fermi surface along three different directions in $k$ space, as a function of carrier density. The dashed line gives the isotropic contribution from the Rashba term, which is seen to be, in this range of carrier concentration, the dominant one in the case of InAs. A small degree of anisotropy appears only at high carrier concentration. The spin split-

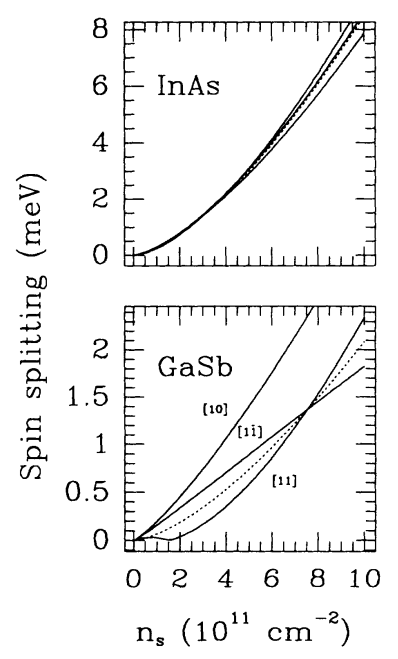

FIG. 3. Zero-field spin splitting at three points of the Fermi surface along different directions in $k$ space (indicated in the case of $\mathrm{GaSb}$ ), as a function of the carrier density $n_{s}$. The dashed line gives the isotropic contribution of the Rashba term alone. ting in GaSb-based heterojunctions is found to be somewhat smaller but much more anisotropic.

The anisotropy in the zero-field spin splitting of both $\mathrm{GaSb}$ - and InAs-based heterojunctions is not simply due to the $k^{3}$ term as assumed in Refs. 24 and 26, for the case of GaAs quantum wells. Though the Rashba term is spherically symmetric, a contribution to the anisotropy originates from the interference between the $k^{3}$ and the Rashba terms; this is responsible, for instance, for the broken symmetry between directions [11] and [11] , as can be seen in Fig. 3.

The zero-field spin splitting manifests itself more clearly producing the already-mentioned beating pattern in the amplitude of the magneto-oscillations; the quantummechanical description of which starts with the problem of the electronic states in a magnetic field.

\section{B. States in a perpendicular magnetic field}

We turn now to the problem of the electronic states when a magnetic field perpendicular to the interface is applied. The stationary states are the eigenstates of the Hamiltonian operator obtained from the one at zero field by simply adding the Zeeman term and substituting everywhere $\mathbf{k} \rightarrow-i \nabla+(e / \hbar) \mathbf{A}$, where $\mathbf{A}$ is the vector potential of the applied field. ${ }^{27}$ In the present case, starting from the $8 \times 8$ Hamiltonian (3) with the addition of the magnetic-field contribution, we carry out the same procedure as before and project on the conduction states to obtain the following Hamiltonian:

$$
H=\left(\begin{array}{cc}
\varepsilon(k)+\frac{1}{2} \mu g^{*}(k) B & \gamma \Omega(\mathbf{k})+i \alpha k_{-} \\
\gamma \Omega^{\dagger}(\mathbf{k})-i \alpha k_{+} & \varepsilon(k)-\frac{1}{2} \mu g^{*}(k) B
\end{array}\right),
$$

where $\varepsilon(k)$ is given by (15), $\alpha$ by (13), and a reduced effective $g$-factor operator $g^{*}$ has been introduced to first order in $\delta$,

$g^{*}(k)=g_{0}^{*}\left[1-\frac{2 E_{g}+\Delta}{E_{g}\left(E_{g}+\Delta\right)} \frac{\hbar^{2}}{2 m^{*}}\left[k^{2}+\frac{b^{2}}{4}\right]\right]$,

the bulk band-edge effective $g$ factor ${ }^{28}$ being

$$
g_{0}^{*}=2\left(1-\frac{m_{e}}{m^{*}} \frac{\Delta}{3 E_{g}+2 \Delta}\right) .
$$

Finally, $k_{ \pm}=k_{x} \pm i k_{y}$ and $^{23}$

$$
\Omega(\mathbf{k})=\frac{1}{4}\left(k_{+} k_{-} k_{+}-k_{-}^{3}\right)-k_{+} \frac{b^{2}}{4} .
$$

The usual way to diagonalize Hamiltonians of this type is to make use of an expansion in harmonic-oscillator functions. ${ }^{29-31}$ It is easy to verify that the operators $a^{\dagger}=(l / \sqrt{2}) k_{+}$and $a=(l / \sqrt{2}) k_{-}$, where $l=\sqrt{\hbar / e B}$ is the magnetic length, satisfy $\left[a^{\dagger}, a\right]=1$. It then follows that they generate a complete set of functions satisfying $a^{\dagger} \phi_{n}=\sqrt{n+1} \phi_{n+1}$ and $a \phi_{n}=\sqrt{n} \phi_{n-1}$, with $\phi_{0}$ being given by $a \phi_{0}=0$. The eigenfunctions of $H$ can then be expanded as: 


$$
\psi=\sum_{n}\left(\begin{array}{l}
c_{n \uparrow} \\
c_{n \downarrow}
\end{array}\right) \phi_{n} .
$$

The matrix elements of $H$ in this basis can be easily calculated $^{6}$ and the eigenvalues are obtained, for every value of $B$, by diagonalizing numerically a sufficiently large matrix to obtain convergence on the lowest states. The convergence in our case, for the number of Landau levels needed to accommodate all the electrons, was reached using a maximum number of basis functions of around 300 in the low field and high $n_{s}$ limit. Figure 4 shows a typical fan diagram with the first few Landau levels. As compared to usual fan diagrams, it is interesting to note, besides the overall nonparabolic bending, a structure of smooth anticrossings at small fields (see inset), which is due to the spin-orbit coupling in the presence of the above-described asymmetries.

\section{RESULTS AND DISCUSSIONS}

\section{A. Oscillations in the magnetization}

Besides the Shubnikov-de Haas measurements mentioned above, there have been also investigations on magnetothermal oscillations of the two-dimensional electron $\operatorname{gas}^{32}$ (2DEG). We have chosen to look at the analogous de Haas-van Alphen oscillations in order to study the beating pattern in the magneto-oscillations of $2 \mathrm{DEG}$, because the magnetization can be calculated in a more direct way than the resistivity. ${ }^{33}$ Oscillations in the magnetization of a 2DEG have been first measured by Störmer et al. ${ }^{34} \mathrm{We}$ consider the magnetization at zero temperature because the experiments are done at very low temperatures, but our reasoning would not be modified by taking into account the statistical distribution. The magnetization (perpendicular to the interface) is given by

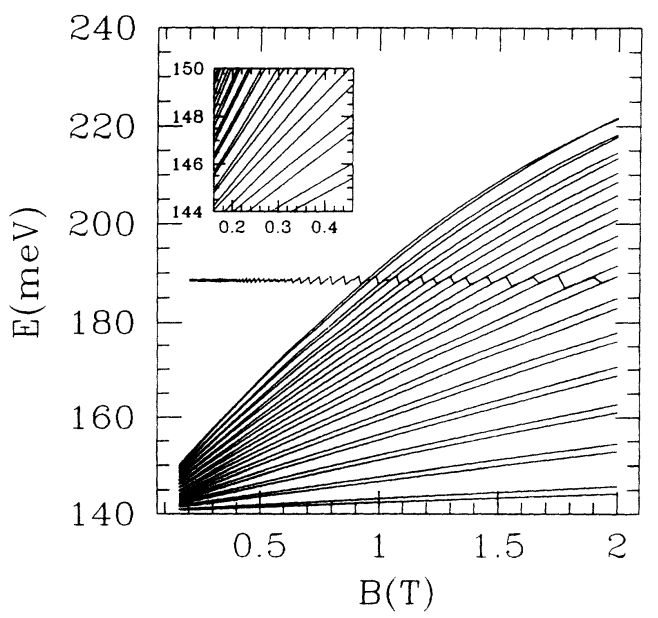

FIG. 4. Fan diagram for an InAs heterojunction with $n_{s}=6.0 \times 10^{11} \mathrm{~cm}^{-2}$. Shown is also the oscillating Fermi energy. The inset shows a detail of the low-field limit with smooth anticrossings.

$$
M=-\left.\frac{d E_{T}}{d B}\right|_{n_{s}},
$$

where

$$
E_{T}=\int_{-\infty}^{\varepsilon_{F}(B)} \varepsilon D_{B}(\varepsilon) d \varepsilon
$$

is the total energy of the 2DEG. The dependence on the magnetic field comes both from the Fermi energy $\varepsilon_{F}$ and from the density of states $D_{B}(\varepsilon)$, which, by assuming a Gaussian broadening of the Landau levels, reads

$$
D_{B}(\varepsilon)=\frac{g / 2}{\sqrt{2 \pi} \Gamma_{B}} \sum_{n} e^{\left[\varepsilon-\varepsilon_{n}(B)\right]^{2} / 2 \Gamma_{B}^{2}},
$$

where the sum is over all spin-resolved discrete levels and $g$ is the degeneracy of the Landau levels. The broadening width is taken field dependent and of the form $\Gamma_{B}=\Gamma_{0} \sqrt{B}$, where $\Gamma_{0}$ is a parameter which depends on the quality of the sample. ${ }^{35}$ Finally, the Fermi energy is determined from:

$$
n_{s}=\int_{-\infty}^{\varepsilon_{F}(B)} D_{B}(\varepsilon) d \varepsilon
$$

Figure 5 shows a typical example of how the total energy, the Fermi energy, and the magnetization vary with the magnetic field. The oscillations in the total energy and in the magnetization follow the oscillations in the Fermi energy. A few Landau levels are also plotted. The Zeeman splitting shows up only in the high-field limit. The beatings are due to the zero-field spin splitting, which can be studied by Fourier analyzing the magnetooscillations as functions of $1 / B$.

The magnetization as a function of $1 / B$ presents periodic-modulated oscillations. In Fig. 6, we have plotted, together with their power spectrum, the oscillations

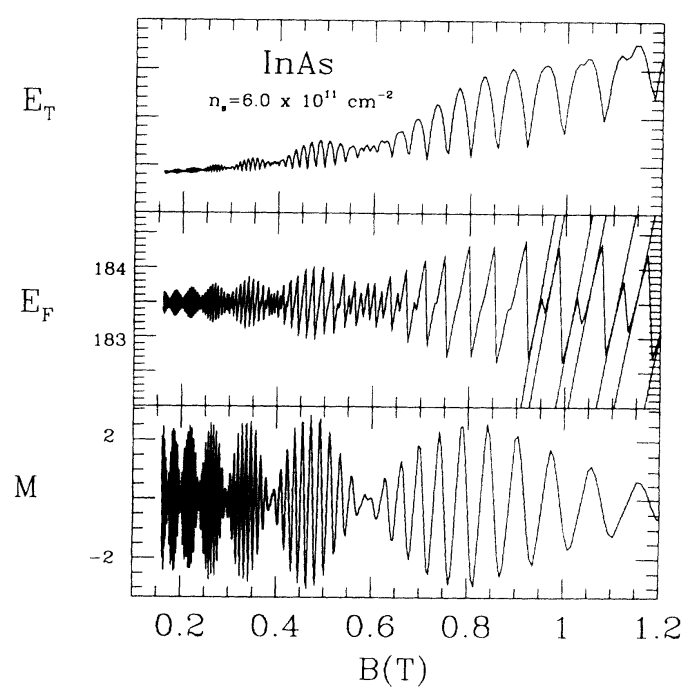

FIG. 5. Typical result for the variation of the total energy $E_{T}$ (in arbitrary units), the Fermi energy $E_{F}$ (in $\mathrm{meV}$ ), and the magnetization $M$ (in units of $10^{11} \mathrm{meV} / \mathrm{T} \mathrm{cm}$ ) as a function of the applied magnetic field $B$. A few Landau levels are also shown $\left(\Gamma_{0}=0.1 \mathrm{meV}\right)$. 


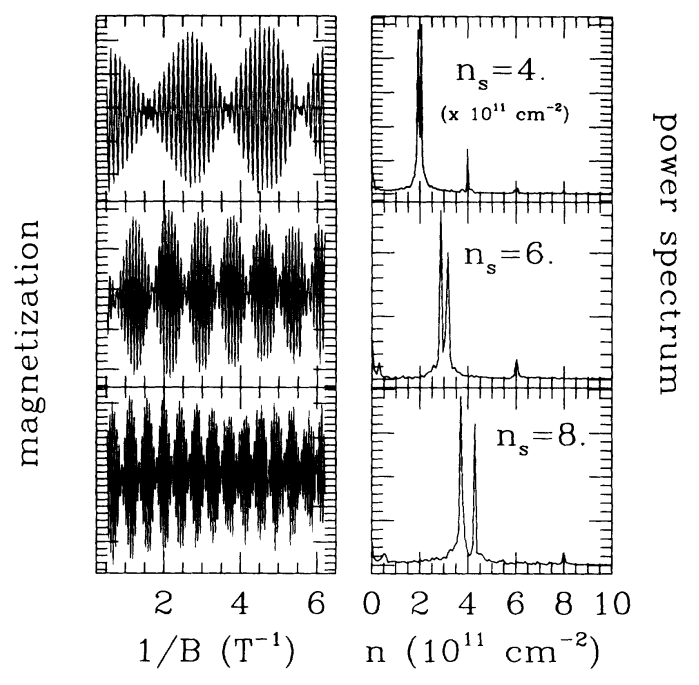

FIG. 6. Oscillating magnetization of a 2DEG at the interface of an InAs-based heterojunction with different carrier concentration $n_{s}\left(4,6\right.$, or $\left.8 \times 10^{11} \mathrm{~cm}^{2}\right)$. On the left panel, the beating pattern is clearly evident. On the right panel, we plot the respective power spectrum in terms of surface density $n$. The oscillation frequencies are shown as strong peaks. They are close doublets, the sum of the two corresponding to $n_{s}$, and higher harmonics.

obtained for InAs heterojunctions with different carrier densities. The power spectrum is the absolute value squared of the Fourier transform of the magnetization and is shown in units of surface densities; as given from the semiclassical expression $v=n_{s} \hbar \pi / e$, the frequency in Teslas is $v[\mathrm{~T}]=4.13 n_{s}\left[10^{11} \mathrm{~cm}^{-2}\right]$. One should first note that the oscillations in $1 / B$, within this range of carrier concentration, present a quite regular beating pattern. The power spectrum shows two near frequencies corresponding to the total number of carriers, which occupy the two spin-split subbands. Such frequencies occur at

$$
n_{ \pm}=\frac{1}{(2 \pi)^{2}} \int d \mathbf{k} \Theta\left[\varepsilon_{F}-\varepsilon_{ \pm}(\mathbf{k})\right]
$$

$[\Theta(x)$ is the Heaviside function], which are the densities in the split bands. Their separation produces the beatings, which increase with total carrier density as a result of the increasing spin splitting at the Fermi energy, as shown in Fig. 3.

The almost isotropic spin splitting, dominated by the Rashba term, in the case of InAs, explains the obtained regular beating pattern. Anomalous beating patterns, due to magnetic breakdown at the points with a smaller spin splitting, are expected when the spin splitting is anisotropic. With increasing $n_{s}$, the oscillations become in fact more irregular as the degree of anisotropy increases, as can be observed in Fig. 6 .

The expected oscillations in the case of heterojunctions made with GaSb help us to understand the relation between anisotropy in the spin splitting and anomalous beatings in the amplitude of the magneto-oscillations. The spin splitting in the case of GaSb (see Fig. 3) is much more anisotropic. It is, however, also somewhat smaller than for InAs and in this range of magnetic fields it leads to only one beat.

We can increase the number of beats by applying an external electric field along the growth direction, thus increasing the contribution of both splitting terms, still preserving a high degree of anisotropy. Figure 7 shows the calculated oscillations for a $n_{s}=6.0 \times 10^{11} \mathrm{~cm}^{-2}$ in $\mathrm{GaSb}$ heterojunction with zero and $50 \mathrm{kV} / \mathrm{cm}$ of applied field. The oscillations are clearly characterized by anomalous beating patterns. The separate peak in the power spectrum, which is seen to build up at $n_{s} / 2$, correspond to magnetic breakdown at the small spin-splitting point along the [11] direction. A richer structure, with up to five peaks in the power spectrum, is seen to appear when using a bulk parameter $\gamma$ twice as big. For GaAs, the values of $\gamma$ quoted in the literature vary by almost a factor of 2. Figure 7 shows that the power spectrum of the magneto-oscillations is very sensitive to $\gamma$, and this may be of help in its experimental determination. We would also like to mention that the power spectrum in the case of anomalous beating pattern depends on the range of magnetic fields.

The only (free) parameter in our calculations is the Landau-level broadening parameter $\Gamma_{0}$. The magnetooscillations are very sensitive to $\Gamma_{0}$. The few oscillations at high magnetic fields observed in Ref. 34 are reproduced well here with $\Gamma_{0}=1.2 \mathrm{meV}$. The obtained amplitude with the nominal carrier concentration is only a factor of 2 larger, which is probably due to the distribution in carrier concentration. ${ }^{34}$ We show in Fig. 8 how the magneto-oscillations for an InAs heterojunction evolve with increasing Landau-level broadening. One can see that, as expected, the oscillations present a decreasing amplitude and are broadened out at small fields. The cal-

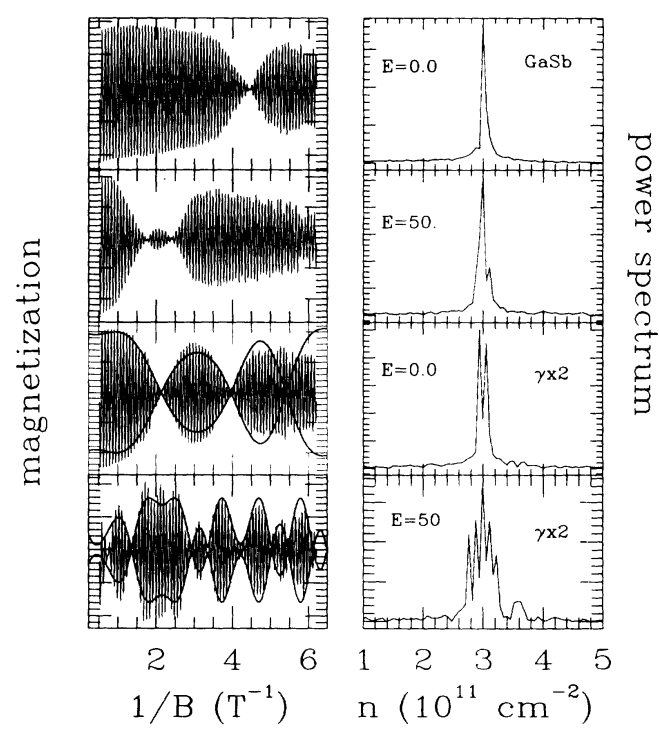

FIG. 7. Anomalous magneto-oscillations for a $n_{s}=6.0 \times 10^{11}$ $\mathrm{cm}^{-2} \mathrm{GaSb}$ heterojunction under zero and $50 \mathrm{kV} / \mathrm{cm}$ of applied electric fields. The parameters are as given in Table II. In the two lower panels, we have used a value of the material parameter $\gamma$ twice as big and have traced with envelope curves the semiclassical fittings (see Sec. III B). 

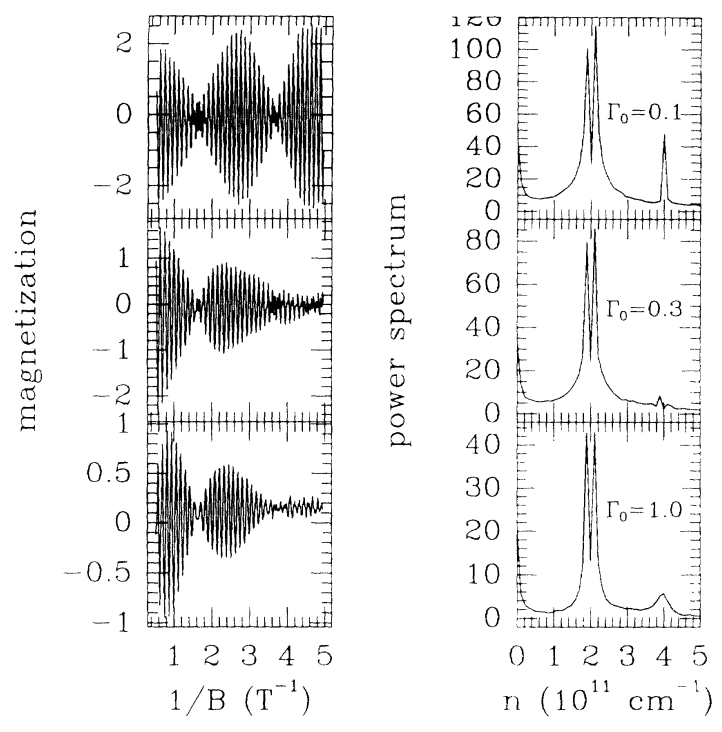

FIG. 8. Effects of the Landau-level broadening on the magneto-oscillations. Magneto-oscillations of a $n_{s}=4.0 \times 10^{11}$ $\mathrm{cm}^{-2}$ InAs heterojunction with three different values of the parameter $\Gamma_{0}$ (in $\mathrm{meV}$ ).

culated oscillations with $\Gamma_{0}=1.0 \mathrm{meV}$ reported in Fig. 8 are quite similar in shape to the observed ones in an InAs asymmetric quantum well with nearly the same carrier concentration. $^{5}$ The split peaks in the power spectrum remain always well defined in this range of broadening parameter, though their amplitude is reduced with increasing broadening. The multipeak structure in the power spectrum connected with the anomalous beating pattern here obtained is, therefore, expected to survive at larger broadenings.

\section{B. Semiclassical analysis}

The anomalous beating pattern obtained above can be rationalized in terms of a semiclassical picture allowing for magnetic breakdown. ${ }^{4,36}$ The semiclassical analysis relates the frequencies of the magneto-oscillations to the areas in $k$ space occupied by the electron gas in zero field (see Fig. 9) and the probabilities of magnetic breakdown to the shape of the Fermi-surface sheets at the points where the spin splitting is smaller (breakdown junctions).

We choose for analytical convenience a model that illustrates the semiclassical approach for a twodimensional electron gas. Let the density of states (per spin per unit area) in the presence of a magnetic field be given by

$D_{B}(\epsilon)=\frac{m^{*}}{2 \pi \hbar^{2}}\left[1+\alpha \cos \left[\frac{2 \pi m^{*}}{\hbar e B} \epsilon-\pi\right)\right] \Theta(\epsilon)$,

where a parameter $\alpha(0<\alpha<1)$ describes the broadening of the Landau levels and all complications such as nonparabolicity and spin splitting are omitted. Then, the Fermi energy $\epsilon_{F}(B)$ is given in terms of $n_{s}$ by

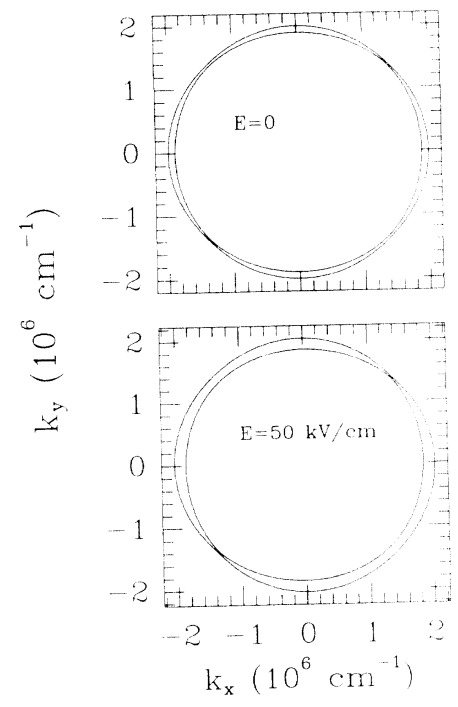

FIG. 9. The 2D Fermi surfaces for the GaSb heterojunctions corresponding to the lower two panels of Fig. 7. The two curves correspond to the two spin-split subbands and give the two sheets of the Fermi surface.

$$
\begin{aligned}
n_{s} / 2 & =\int_{0}^{\epsilon_{F}(B)} D_{B}(\epsilon) d \epsilon \\
& =\frac{e B}{(2 \pi)^{2} \hbar}\{F(B)-\alpha \sin [F(B)]\},
\end{aligned}
$$

where

$$
F(B)=\frac{2 \pi m^{*}}{\hbar e B} \epsilon_{F}(B) .
$$

From Eqs. (25) and (26), the magnetization can be written as

$$
\begin{gathered}
M(B)=-\epsilon_{F}(B) D_{B}\left[\epsilon_{F}(B)\right] \frac{d \epsilon_{F}(B)}{d B}-\int_{0}^{\epsilon_{F}(B)} \epsilon \frac{d D_{B}(\epsilon)}{d B} d \epsilon \\
=-\frac{\alpha e^{2} B}{(2 \pi)^{3} m^{*}}\{2-2 \cos [F(B)] \\
\quad-F(B) \sin [F(B)]\} .
\end{gathered}
$$

Since the semiclassical expression for the oscillating magnetization is

$$
M_{\mathrm{SC}}(B)=\frac{e \epsilon_{F}}{2 \pi^{2} \hbar} \sin \left(\frac{\hbar A\left(\epsilon_{F}\right)}{e B}\right),
$$

where $A\left(\epsilon_{F}\right)$ is the area in $k$ space filled by the 2D Fermi sea in zero field, given by

$$
A=\frac{2 \pi m^{*}}{\hbar^{2}} \epsilon_{F}
$$

using expression (32), we obtain

$$
M_{\mathrm{SC}}(B)=\frac{e^{2} B}{4 \pi^{3} m^{*}} F(B) \sin [F(B)],
$$

where $F(B)$ can be computed from expression (31). In the limit of large quantum numbers, $F \gg 1$ and $M_{\mathrm{SC}}$ 
agrees with $M$ (apart from a numerical factor of order unity); in the same limit, the magneto-oscillations are approximately given by

$$
M_{\mathrm{SC}} \simeq \frac{e \hbar n_{s}}{2 \pi m^{*}} \sin \left(\frac{(2 \pi)^{2} \hbar n_{s}}{2 e B}\right),
$$

which is the $2 \mathrm{D}$ version of the Onsager expression discussed in the Introduction.

In our case, because of the spin splitting, the Fermi surface has two sheets that come close together both at two equivalent points along the [11] and [ $\overline{11}]$ directions and at two equivalent points along the [11] and [11] directions (see Fig. 9). At such breakdown junctions, where the spin splitting is smaller, an electron moving along a semiclassical orbit may tunnel from one sheet of the Fermi surface to the other. To calculate the magneto-oscillations, all possible ways in which an electron can complete one turn (switching or not from one sheet to the other at the four junctions) are to be combined. At each magnetic-breakdown junction, there is a probability amplitude $p=e^{-W / 2 B}$ for the electron to tunnel and $q=i\left(1-p^{2}\right)^{1 / 2}$ for the electron to remain on the same sheet, where $W$ is the so-called magneticbreakdown field, ${ }^{10}$ which can be roughly estimated by

$$
W=\frac{\pi \hbar}{e} \sqrt{(\Delta k)^{3} /(a+b)},
$$

where $\Delta k$ is the distance in $k$ space between the two Fermi-surface sheets at the junction, and $1 / a$ and $1 / b$ are their radii of curvature. The anomalous beating pattern, which gives the envelope of the fast oscillation expressed by Eq. (37), is described by the so-called magneticbreakdown function ${ }^{4,36} G(B)$, which in our case is given by

$$
\begin{aligned}
G(B)= & q_{1}^{2} q_{2}^{2} \cos \left(\frac{2 \hbar \Delta}{e B}-\delta\right) \\
& +4 p_{1} p_{2} q_{1} q_{2} \cos \left(\frac{\hbar \Delta}{e B}-\delta / 2\right) \\
& +\left(p_{1}^{2} p_{2}^{2}+p_{1}^{2} q_{2}^{2}+p_{2}^{2} q_{1}^{2}\right),
\end{aligned}
$$

where $p_{1}, q_{1}\left(p_{2}, q_{2}\right)$ are the above-defined $B$-dependent amplitudes for the two couples of breakdown junctions, $4 \Delta$ is the difference between the $k$-space areas of the outer and inner sheets of the Fermi surface, and $2 \delta$ is the difference between their Onsager phases. The first term on the right-hand side of Eq. (39) corresponds to the beating between the orbit that always stays on the outer sheet and the orbit that always stays on the inner one; the second term corresponds to the beating between the four orbits that stay for $\frac{3}{4}$ of a turn on the outer sheet and for $\frac{1}{4}$ of a turn on the inner one and the four orbits that stay for $\frac{1}{4}$ of a turn on the outer sheet and for $\frac{3}{4}$ of a turn on the inner one; the last term corresponds to the six orbits that stay for $\frac{1}{2}$ of a turn on the outer sheet and for $\frac{1}{2}$ of a turn on the inner one. In Fig. 7, the thick solid lines show the fit to the anomalous beating patterns obtained from the semiclassical analysis embodied in $G(B)$. The parameters used are listed in Table III along with their
TABLE III. Fitting parameters used in the semiclassical analysis of the anomalous beating patterns shown in Fig. 7; in parentheses are the corresponding values estimated from the Fermi "surface" geometry (see text).

\begin{tabular}{cclll}
\hline \hline$E(\mathrm{kV} / \mathrm{cm})$ & $4 \Delta \hbar / e(\mathrm{~T})$ & $W_{1}(\mathrm{~T})$ & $W_{2}(\mathrm{~T})$ & $\delta / \pi$ \\
\hline 0.0 & $6.71(5.72)$ & $0.064(0.12)$ & $0.18(0.29)$ & 3.06 \\
30 & $10.15(8.84)$ & $0.074(0.01)$ & $0.62(1.3)$ & 0.33 \\
50 & $11.95(10.92)$ & $0.21(0.07)$ & $0.94(2.2)$ & 3.98 \\
\hline \hline
\end{tabular}

values estimated from the Fermi-surface geometry (the phase difference $2 \delta$ cannot be readily evaluated). Thus, the semiclassical analysis allowing for magnetic breakdown provides a good interpretation of complex anomalous beating patterns.

\section{Comments on other spin-splitting measurements}

The beating pattern in the amplitude of the magnetooscillations have been studied here as the main probe of the zero-field spin splitting. Other kinds of measurements in semiconductor heterostructures have recently been explained with the zero-field spin splitting. Ramanscattering measurements of intrasubband excitations have shown spin-flip single-particle transitions between spinsplit subbands of an asymmetric modulation-doped $\mathrm{GaAs} / \mathrm{Al}_{0.33} \mathrm{Ga}_{0.67} \mathrm{As}$ quantum well. ${ }^{26}$ Anisotropy in the spin splitting has also been detected. The calculated spectra with only the bulk $k^{3}$ term showed, however, poor agreement with experiment. ${ }^{26}$

The other recent experimental probe of the zero-field spin splitting is connected with the phenomenon of antilocalization. The spin dephasing responsible for the observed negative magnetoresistance (due to antilocalization) has been shown to be due to the precession of the electron spin around the spin-splitting effective magnetic field. ${ }^{37,38}$ The spin dephasing rate in this case follows the following motional narrowing low: ${ }^{22}$

$$
t_{s}^{-1}=a \frac{\left\langle\Delta_{s}^{2}\right\rangle}{4 \hbar^{2}} t_{t}
$$

where \langle\rangle means average over the Fermi surface, $t_{t}$ is the transport (or elastic) scattering time, and $a$ is a parameter of the order of unit that depends on the scattering mechanism. The average spin splitting $\left\langle\Delta_{s}^{2}\right\rangle$ at the Fermi energy has then been estimated for different heterostructrues. ${ }^{37-39}$ Even though our model is not expected to give an accurate value for the Rashba coupling parameter in the case of GaAs (where interaction with higher conduction bands should not be neglected ${ }^{13}$ ), we compare in Fig. 10 the computed values of $\left\langle\Delta_{s}^{2}\right\rangle$ using our procedure with the experimental values obtained by Dresselhaus et al. ${ }^{38}$ from fitting of magnetoresistance measurements in GaAs inversion layers. We can see that the observed dependence on $n_{s}$ is better reproduced when including both $k^{3}$ and Rashba contributions. We have checked that the $k^{3}$ term alone with any pair of parameters $\gamma$ and $a$, within their uncertainties, is not able to give a good fit of the experiment; in Fig. 10, we have used $\gamma=17 \mathrm{eV} \AA^{3}$ and $a=0.5$. This is in agreement with recent criticisms 


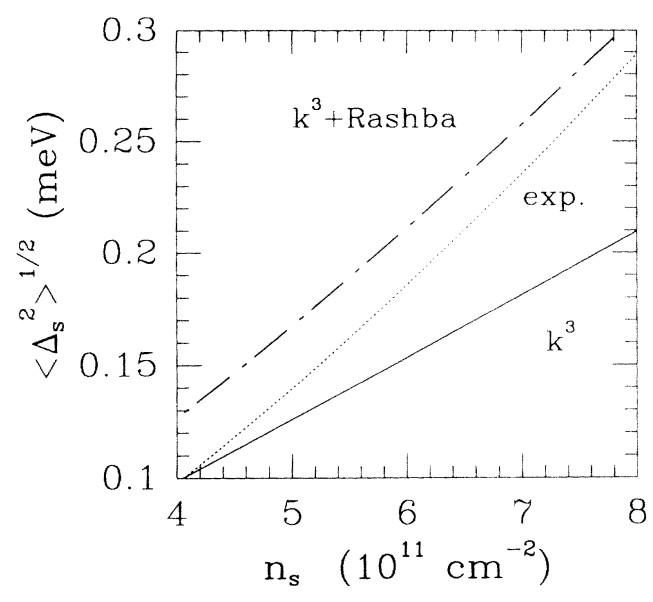

FIG. 10. Comparison of the computed average total spin splitting with that obtained from Dresselhaus et al. ${ }^{38}$ antilocalization data in $\mathrm{GaAs} / \mathrm{Ga}_{1-x} \mathrm{Al}_{x}$ As inversion layers using $a=0.5$. Two theoretical cases are shown; one with both contributions to the spin splitting and one with only the $k^{3}$. The parameters used were $\gamma=17 \mathrm{eV} \AA^{3}, m^{*}=0.067 m_{e}, E_{g}=1.52 \mathrm{eV}$, $\Delta=0.341 \mathrm{eV}$, and $\epsilon_{\mathrm{SC}}=12.85$.

of the original explanation by Santos and Cardona. ${ }^{25}$

The usually neglected Rashba contribution to the spin splitting in GaAs inversion layers has an increasing importance with increasing carrier concentration. We believe that it should also be invoked in order to completely understand the experimental results in Ref. 38. One can see from Fig. 10 that the bigger contribution from the Rashba term at higher values of $n_{s}$ tends to bend up the curve getting it closer to the experiment. We have also compared our results for an InSb inversion layer with the experiment of Greene et al. ${ }^{37}$ and have obtained good agreement.

Finally, the understanding of the spin-dependent electronic properties of these heterostructures is important in the interpretation of time-dependent and polarizationresolved optical measurements.

\section{CONCLUSIONS}

We may summarize our results as follows. We have found the conduction states in asymmetric heterostructures from an eight-band model, taking full account of the spin-orbit interaction, and including two types of spin-splitting contributions, one due to bulk inversion asymmetry and the other to the absence of specular symmetry.

Detailed calculations have been carried out for heterojunctions of InAs and GaSb with larger band-gap materials for different electron densities. The resulting spin splitting is shown to be strongly dependent on carrier concentration and to be anisotropic, the amount of anisotropy depending on both splitting contributions.

We have computed the magnetization and its power spectrum as functions of an external magnetic field. The de Haas-van Alphen oscillations are shown to display clear beating patterns corresponding to two main resonance frequencies associated to the two spin-split subbands. We find anomalies in the beating patterns due to the anisotropy in the spin splitting; their dependence on carrier density and on the confining potential is shown.

The quantum-mechanical results are interpreted using a semiclassical analysis with magnetic breakdown at the points of the Fermi surface with small spin splitting.

Other experimental probes of the spin splitting have also been discussed for the case of $\mathrm{GaAs} / \mathrm{Ga}_{1-x} \mathrm{Al}_{x} \mathrm{As}$ heterojunctions, and the importance of considering both contributions to the spin splitting of the conduction subbands has been suggested.

\section{ACKNOWLEDGMENTS}

One of the authors (E.A.S.) wishes to acknowledge the support of the CNPq-Brazil and of the Saint Gobain Company, which made this research possible.
${ }^{*}$ Permanent address: Instituto Nacional de Pesquisas Espaciais, CP515, 12201 São José dos Campos, Săo Paulo, Brazil.

${ }^{1}$ G. Dresselhaus, Phys. Rev. 100, 580 (1955).

${ }^{2}$ D. G. Seiler, W. M. Becker, and L. M. Roth, Phys. Rev. B 1, 764 (1970); Y.-F. Chen, M. Dobrowolska, J. K. Furdyna, and S. Rodriguez, ibid. 32, 890 (1985); M. M. Miller and R. Reifenberger, ibid. 38, 3423 (1988).

${ }^{3}$ L. M. Roth, Phys. Rev. 173, 755 (1968).

${ }^{4}$ G. C. La Rocca, M. M. Miller, and S. Rodriguez, Phys. Rev. B 40, 11723 (1989).

5J. Luo, H. Munekata, F. F. Fang, and P. J. Stiles, Phys. Rev. B 38, 10142 (1988); 41, 7685 (1990).

${ }^{6}$ B. Das, D. C. Miller, S. Datta, R. Reifenberger, W. P. Hong, P. K. Bhattacharya, J. Singh, and M. Jaffe, Phys. Rev. B 39, 1411 (1989); B. Das, S. Datta, and R. Reifenberger, ibid. 41, 8278 (1990).

${ }^{7}$ Yu. L. Ivanov, P. Kop'ev, S. Suchalkin, and V. Ustinov, Pis'ma Zh. Eksp. Teor. Fiz. 53, 470 (1991) [JETP Lett. 53, 493 (1991)].

${ }^{8}$ Yu. L. Bychkov and E. I. Rashba, Pis'ma Zh. Eksp. Teor. Fiz.
39, 66 (1984) [JETP Lett. 39, 78 (1984)]; J. Phys. C 17, 6039 (1984).

${ }^{9}$ L. Landau, Z. Phys. 64, 629 (1930).

${ }^{10} \mathrm{D}$. Shoenberg, Magnetic Oscillations in Metals (Cambridge University Press, Cambridge, England, 1984).

${ }^{11}$ A. B. Fowler, F. F. Fang, W. E. Howard, and P. J. Stiles, Phys. Rev. Lett. 16, 901 (1966).

${ }^{12}$ L. Onsager, Philos. Mag. 43, 1006 (1952).

${ }^{13}$ G. Lommer, F. Malcher, and U. Rössler, Phys. Rev. Lett. 60, 728 (1988).

${ }^{14}$ E. A. de Andrada e Silva, Phys. Rev. B 46, 1921 (1992).

${ }^{15}$ E. O. Kane, J. Phys. Chem. Solids 1, 249 (1957).

${ }^{16}$ G. E. Marques and L. J. Sham, Surf. Sci. 113, 131 (1982).

${ }^{17}$ M. Cardona, N. E. Christensen, and G. Fasol, Phys. Rev. B 38, 1806 (1988).

${ }^{18}$ A. T. Gorelenko, B. A. Marushchak, and A. N. Titkov, Izv. Akad. Nauk SSSR, Ser. Fiz. 50, 290 (1986).

${ }^{19} \mathrm{~A}$ similar expansion was first used in G. Brozak, E. A. de Andrada e Silva, L. J. Sham, F. DeRosa, P. Miceli, S. Schwarz, 
J. P. Harbison, L. T. Flores, and S. J. Allen, Phys. Rev. Lett. 64, 471 (1990).

${ }^{20}$ G. Bastard, Wave Mechanics Applied to Semiconductor Heterostructures (Les Editions de Physique, Les Ulis, 1990).

${ }^{21}$ For a review, see, G. E. Pikus, V. A. Marushchak, and A. N. Titkov, Fiz. Tekh. Poluprovodn. 22, 185 (1988) [Sov. Phys. Semicond. 22, 115 (1988)].

${ }^{22}$ M. I. D'yakonov and V. I. Perel, Zh. Eksp. Teor. Fiz. 60, 1954 (1971) [Sov. Phys. JETP 33, 1053 (1971)].

${ }^{23}$ N. Kim, G. La Rocca, S. Rodriguez, and F. Bassani, Riv. Nuovo Cimento 12, 1 (1989).

${ }^{24}$ R. Eppenga and M. F. H. Schuurmans, Phys. Rev. B 37, 10923 (1988).

${ }^{25}$ P. V. Santos and M. Cardona, Phys. Rev. Lett. 72, 432 (1994).

${ }^{26}$ D. Richards, B. Jusserand, H. Peric, and B. Etienne, Phys. Rev. B 47, 16028 (1993).

27J. M. Luttinger and W. Kohn, Phys. Rev. 97, 869 (1955).

${ }^{28}$ L. M. Roth, B. Lax, and S. Zwerdling, Phys. Rev. 114, 90 (1959).

${ }^{29}$ S.-R. Eric Yang, D. A. Broido, and L. J. Sham, Phys. Rev. B 32, 6630 (1985).

${ }^{30}$ Yu. A. Bychkov, V. I. Mel'nikov, and E. I. Rashba, Zh. Eksp. Teor. Fiz. 98, 717 (1990) [Sov. Phys. JETP 71, 401 (1990)].
${ }^{31}$ V. E. Bisti and V. I. Fal'ko Fiz. Tverd Tela (St. Petersburg) 34, 1590 (1992) [Sov. Phys. Solid State 34, 839 (1992)].

${ }^{32}$ W. Zawadzki and R. Lassnig, Solid State Commun. 50, 537 (1984).

33J. Hu and A. H. MacDonald, Phys. Rev. B 46, 12554 (1992).

${ }^{34}$ H. L. Störmer, T. Haavasoja, V. Narayanamurti, A. C. Gossard, and W. Wiegmann, J. Vac. Sci. Technol. B 1, 423 (1983); T. Haavasoja, H. L. Störmer, D. J. Bishop, V. Narayanamurti, A. C. Gossard, and W. Wiegmann, Surf. Sci. 142, 294 (1984).

${ }^{35} \mathrm{R}$. Lassing and E. Gornik, in Two-Dimensional Systems: Physics and New Devices, edited by G. Bauer, F. Kucher, and H. Heinrich, Springer Series in Solid-State Sciences Vol. 67 (Springer-Verlag, Berlin, 1987).

${ }^{36}$ R. Reifenberger and D. A. Schwarzkopf, Phys. Rev. Lett. 50, 907 (1983)

${ }^{37}$ S. K. Greene, J. Singleton, P. Sobkowicz, T. D. Golding, M. Pepper, J. A. Perenboom, and J. Dinan, Semicond. Sci. Technol. 7, 1377 (1992).

${ }^{38}$ P. D. Dresselhaus, C. M. Papavassiliou, R. G. Wheeler, and R. N. Sacks, Phys. Rev. Lett. 68, 106 (1992).

${ }^{39}$ G. L. Chen, J. Han, T. T. Huang, S. Datta, and D. B. Janes, Phys. Rev. B 47, 4084 (1993). 

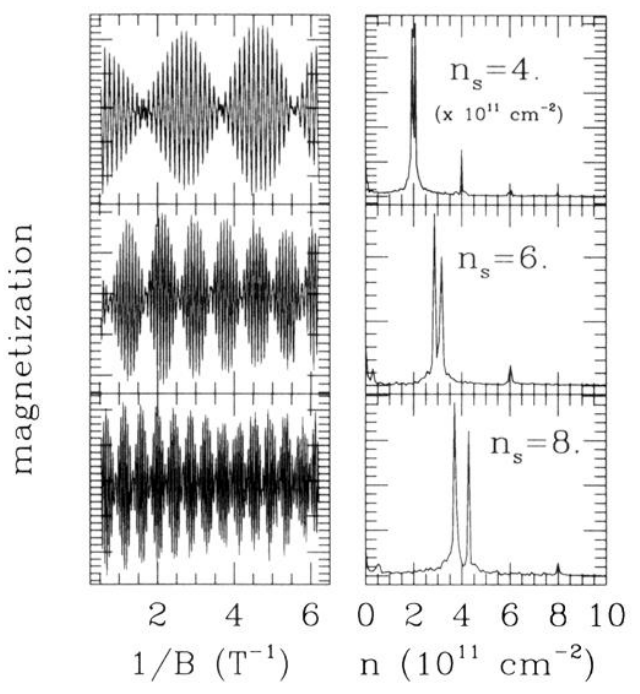

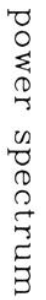

FIG. 6. Oscillating magnetization of a $2 \mathrm{DEG}$ at the interface of an InAs-based heterojunction with different carrier concentration $n_{s}\left(4,6\right.$, or $\left.8 \times 10^{11} \mathrm{~cm}^{2}\right)$. On the left panel, the beating pattern is clearly evident. On the right panel, we plot the respective power spectrum in terms of surface density $n$. The oscillation frequencies are shown as strong peaks. They are close doublets, the sum of the two corresponding to $n_{s}$, and higher harmonics. 


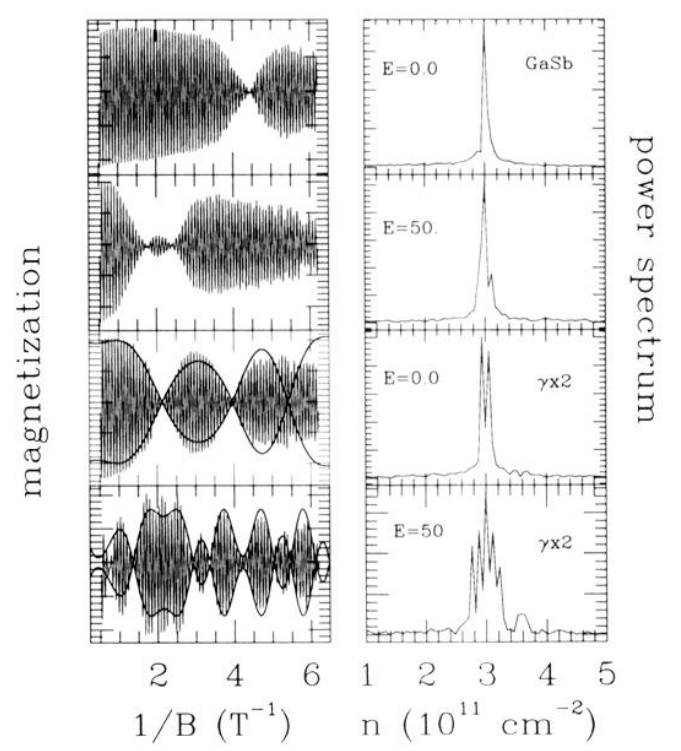

FIG. 7. Anomalous magneto-oscillations for a $n_{s}=6.0 \times 10^{11}$ $\mathrm{cm}^{-2} \mathrm{GaSb}$ heterojunction under zero and $50 \mathrm{kV} / \mathrm{cm}$ of applied electric fields. The parameters are as given in Table II. In the two lower panels, we have used a value of the material parameter $\gamma$ twice as big and have traced with envelope curves the semiclassical fittings (see Sec. III B). 